\title{
NOUVELLE
}

\section{Le rôle des lymphocytes T régulateurs dans l'activité antitumorale des anticorps anti-CTLA-4}

\author{
Kevin Thierry ${ }^{1}$, Christine Ménétrier-Caux ${ }^{2}$
}

IUniversité Claude Bernard Lyon 1, 69000 Lyon, France.

${ }^{2}$ Université de Lyon, Université Claude Bernard Lyon 1, Inserm U1052, CNRS 5286, Centre Léon Bérard, Centre de Recherches en Cancérologie de Lyon, 69008 Lyon, France. Kevin.thierry@etu.univ-lyonl.fr

Christine.caux@lyon.unicancer.fr

Les anticorps anti-CTLA-4

dans le traitement des cancers décrit à la fin des années 1980 [1], est un récepteur transmembranaire (de 25 $\mathrm{kDa}$ ) qui participe, du fait de sa plus forte affinité pour les récepteurs CD80/ CD86 sur la CPAg, à l'immunosuppression en diminuant indirectement la signalisation via CD28 pendant la présentation de l'Ag. Ceci augmente le seuil d'activation des LT et réduit la réponse aux $\mathrm{Ag}$ de faible affinité. De plus, l'interaction entre CTLA-4 sur le LT et CD80/CD86 sur la CPAg peut entraîner un phénomène de transendocytose qui correspond au transfert de CD80/CD86 de la CPAg vers le $\mathrm{LT}$ réduisant la capacité de la CPAg à stimuler le LT via CD28. Les souris déficientes pour CTLA-4 globalement ou uniquement dans les Treg développent des troubles lymphoprolifératifs qui détruisent les organes vitaux suggérant un rôle central de CTLA-4 dans la tolérance immunitaire.

La présence de Treg dans le microenvironnement tumoral est associée à un mauvais pronostic et à un développement métastatique dans de nombreuses tumeurs solides [2]. CTLA-4, au vu de ses fonctions, a représenté une cible thérapeutique pionnière en immunothérapie [3]. En effet, bloquer le signal CTLA-4 par un anticorps (Ac) permet de restaurer le signal de costimulation et d'activer les LT. Différentes équipes ont rapporté la forte expression par les Treg intratumoraux des principaux ICP, rhéostats qui permettent, en fonction des situations, la régulation positive ou négative de leurs fonctionnalités.

\section{Les modes d'action des anticorps anti- CTLA-4}

Le mode d'action proposé pour expliquer le rejet tumoral induit par les $A c$ anti-CTLA-4 passe par leur capacité à favoriser le «priming » des LT naïfs dans les organes lymphoïdes drainants la tumeur et promouvoir leur prolifération. Des données plus récentes suggèrent des mécanismes alternatifs tels que le blocage de la transendocytose des molécules CD80/CD86 sur les CPAg et surtout la déplétion des Treg dans la tumeur. $\varepsilon n$ effet, en plus de leur activité d'inhibiteurs compétitifs de l'interaction entre CD80/CD86 et CD28, il a récemment été démontré que les $A c$ antiCTLA-4 peuvent induire une déplétion des cellules CTLA-4+ $4^{+}$ar un mécanisme d'ADCC (antibody-dependent cellular cytotoxicity). L'ipilimumab, Ac d'isotype IgGl, développé par James Allison (prix Nobel de physiologie ou médecine en 2018), montre in vitro une capacité de déplétion des Treg CTLA-4 ${ }^{+}$en présence de monocytes et de cellules NK (natural killer) exprimant différents types de récepteurs des IgG (RFc $\gamma)$. Une étude récente montre que la déplétion des Treg dans l'environnement tumoral joue un rôle clef dans l'effet thérapeutique des Ac anti-CTLA-4 [4]. Ces observations sont en accord avec une autre étude réalisée chez la souris qui démontre que, non seulement l'homologue murin de l'ipilimumab 
ne bloque pas la transendocytose de CD80/CD86, mais qu'en plus le blocage de l'interaction CD80/CD86 - CTLA-4 n'est pas nécessaire à une réponse thérapeutique qui dépend majoritairement des RFcy de l'hôte [5].

Ces observations suggèrent que le choix de l'isotype pour ces Ac thérapeutiques anti-ICP est très important puisque qu'une partie du mécanisme d'action implique leur fixation aux RFc $\gamma$.

Deux anticorps thérapeutiques, ipilimumab versus trémélimumab; une efficacité différente?

L'ipilimumab a obtenu l'autorisation de mise sur le marché en 2011 pour le traitement des mélanomes avancés suite à des essais cliniques rapportant une efficacité chez 10 à $20 \%$ des patients avec des réponses cliniques durables. Le traitement par l'ipilimumab est fréquemment associé à des toxicités sévères de type auto-immun dont la prise en charge clinique est aujourd'hui améliorée. Ces manifestations auto-immunes démontrent sur le plan biologique que cet $A c$ affecte probablement le nombre ou la fonctionnalité des Treg. Un autre Ac anti-CTLA-4, le trémélimumab, d'isotype IgG2a a été développé pour diminuer l'activation du complément et les risques de relargage cytokinique liés à la fixation aux RFc $\gamma$. Cependant, même si des réponses ont été observées chez certains patients traités avec le trémélimumab, l'étude de phase III n'a pas démontré d'efficacité supérieure à la chimiothérapie.

Cette différence d'efficacité thérapeutique ne résulte pas de l'épitope reconnu sur CTLA-4 puisque les deux Ac se fixent sur la même région de la molécule avec la même affinité. Néanmoins il est à noter qu'ils diffèrent légèrement dans leurs paratopes et dans leur cinétique de fixation, avec une interaction CTLA-4 - ipilimumab moins stable [6]. Cependant, ces données ne suffisent pas à expliquer les différences cliniques observées qui pourraient résulter de la variation dans la région $\mathrm{Fc}$ des Ac.
Les Ac anti-CTLA-4 entraînent une déplétion des Treg dépendante de la région $\mathrm{Fc}$

La déplétion des Treg par les Ac antiCTLA-4 repose sur un mécanisme d'ADCC dépendant des cellules $R F c \gamma^{+}$du microenvironnement tumoral

Les différences inter-espèces entre l'homme et la souris concernant les récepteurs $R F c \gamma$ rendent difficile l'évaluation de l'implication des mécanismes dépendant de ces récepteurs dans l'activité in vivo des $A c$ thérapeutiques humains [7]. Pour contourner ces difficultés, Arce-Vargas et al. [4], ont utilisé un modèle, développé précédemment, de souris transgéniques exprimant les RFc $\gamma$ humains (hRFc $\gamma$ ).

La comparaison du micro-environnement des tumeurs humaines ou implantées chez la souris hRFc $\gamma$, montre un patron d'expression des hRFc $\gamma$ proche, même si certaines différences non anecdotiques existent : 1) la présence de hRFcyl sur les polynucléaires neutrophiles, les cellules NK et les lymphocytes B (LB) chez la souris hRFcy mais pas chez l'homme; 2) la présence de hRFcyllA sur les cellules NK et les LB dans l'environnement tumoral chez la souris, ce qui n'est pas le cas chez l'homme ; 3) la présence de hRFcyllB sur les monocytes et macrophages uniquement dans l'environnement tumoral chez l'homme alors qu'il est exprimé aussi en périphérie chez la souris.

L'objectif de cette étude était d'évaluer, dans les souris hRFc $\gamma$, l'efficacité anti-tumorale 1) d'anticorps chimériques dirigés contre CTLA-4 de souris et ayant des régions $\mathrm{Fc}$ humaines issues d'IgGl et d'IgG2 humaines et 2) d'IgGl humaines anti-CTLA-4 dont les séquences de la région $\mathrm{Fc}_{c}$ ont été mutées, conduisant soit à une perte de capacité de fixation aux RFc $\gamma \| \mathrm{A}$ et RFc $\gamma \| I \mathrm{~A}\left(\mathrm{hlgG} \mathrm{N}_{\mathrm{N} 297 \mathrm{~A}}\right)$, soit à une affinité accrue pour tous les RFc $\gamma$ (hlgGl $\left.l_{\text {SDALIE }}\right)$.

Cette évaluation a été réalisée dans trois modèles tumoraux murins différentiellement infiltrés en cellules immunitaires: deux «tumeurs chaudes » for- tement infiltrées (MCA205, MC38) et une « tumeur froide » très faiblement infiltrée (B16). Ces tumeurs se différencient aussi par le niveau d'expression des RFc $\gamma$ sur les populations immunitaires innées.

\section{L'efficacité des anticorps anti-CTLA-4 dépend de la région $\mathrm{Fc}$}

Les travaux de Selby et al. [8] ont démontré chez la souris, dans des modèles tumoraux syngéniques (MC38, CT26), que l'efficacité thérapeutique des Ac antiCTLA-4 murins dépend des isotypes utilisés. Les mlgG2a et mlgG2b, qui se fixent sur les RFc $\gamma$ activateurs et induisent une ADCC efficace, favorisent la déplétion des Treg uniquement dans l'environnement tumoral et inhibent la croissance tumorale. Au contraire, les isotypes $\mathrm{mlgGl}$, qui se fixent sur le RFc $\gamma$ inhibiteur, RFc $\gamma \| \mathrm{IB}$, qui n'induit pas d'ADCC, n'impactent pas la croissance tumorale (Figure 1).

Arce-Vargas et al. [4], apportent la preuve, dans le modèle murin $\mathrm{RhFc} \gamma$, que les anti-CTLA-4 d'isotype hlgG2, hlgGl et $\mathrm{hlgG} \mathrm{l}_{\text {SDALIE }}$ déplètent les Treg dans l'environnement tumoral.

L'abrogation de la déplétion observée avec l'isotype hlgG2 dans les souris hRFcylla-déficientes, démontre l'importance de ce récepteur activateur dans l'efficacité de cet isotype. La version $\operatorname{lgG} l_{\text {SDALIE, }}$ qui a une affinité accrue pour le RFcyllIA, accroît fortement l'efficacité du traitement. Ces résultats confirment le concept selon lequel la forte expression membranaire de CTLA-4 par les Treg, spécifiques de l'environnement tumoral, favorise leur élimination par ADCC lors du traitement par les Ac antiCTLA-4. Cette déplétion est associée à une prolifération et une production accrue d'IFN $\gamma$ par les LT CD4 $4^{+}$conventionnels et les $\mathrm{LT} C D 8^{+}$intra-tumoraux, confirmant ainsi la levée de l'immunosuppression dépendante des Treg.

Dans les tumeurs chaudes, l'efficacité thérapeutique des Ac anti-CTLA-4 est directement corrélée au niveau de déplétion des Treg et dépend de l'isotype, alors que dans la tumeur froide, les différents isotypes n'ont 


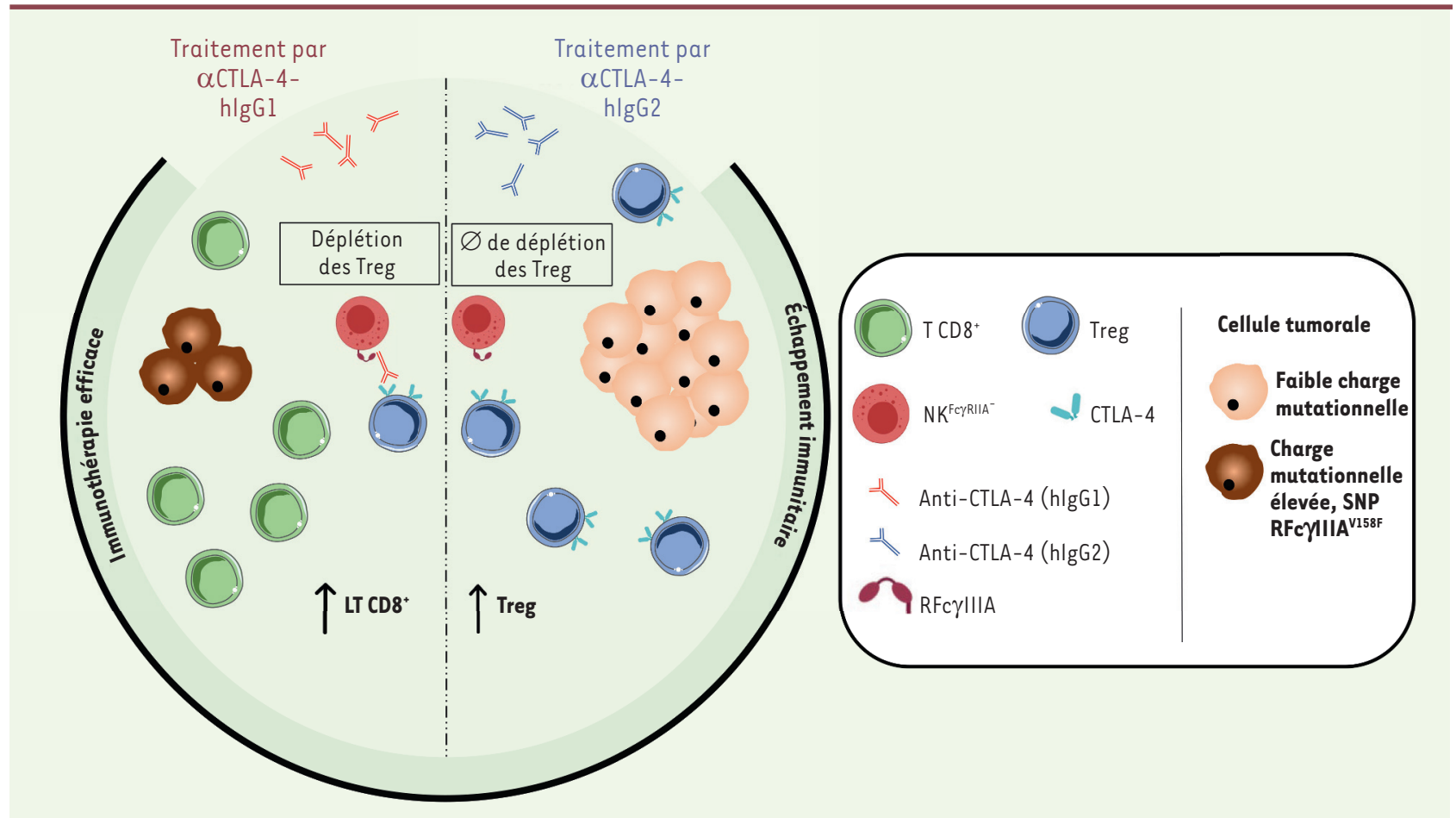

Figure 1. Chez l'homme, l'activité anti-tumorale des anticorps anti-CTLA-4 dépend d'une déplétion des Treg intra-tumoraux par ADCC qui n'est observée qu'avec l'isotype IgG1. L'activité des anti-CTLA-4 est optimale dans un contexte de forte charge mutationnelle chez des patients présentant le SNP RFc $\gamma I I I A^{V 158 F} . \emptyset:$ Absence

aucun impact sur la survie des souris même en utilisant des doses plus fortes d'Ac. Ces observations suggèrent que la réponse thérapeutique des $A c$ anti-CTLA-4 dépend de la déplétion des Treg intra-tumoraux via un mécanisme d'ADCC qui dépend de l'isotype de l'Ac thérapeutique et de l'infiltrat intratumoral de cellules RFc $\gamma^{+}$(en particulier les cellules myéloïdes ou les cellules NK chez la souris) capables d'induire ce type de mécanisme.

\section{L'action des Ac anti-CTLA-4 chez l'homme}

L'activité des Ac anti-CTLA-4 est influencée par un terrain génétique particulier et par la charge mutationnelle tumorale Les polymorphismes mononucléotidiques (SNP) des RFcyllIA (V158F) et RFcyllA (H13lR) observés respectivement dans $10-15 \%$ et $28 \%$ de la population caucasienne, augmentent l'affinité de fixation des hlgGl et des hlgG2 respectivement et sont associés à une meilleure réponse clinique dans le cas d'immunothérapies ciblant les cellules tumorales (rituximab, trastuzumab, cétuximab).

La charge mutationnelle qui correspond au nombre de mutations présentes dans la tumeur, et les néo-antigènes, conséquences de mutations passagères nononcogéniques ont été identifiés comme des marqueurs prédictifs de réponse à l'ipilimumab dans le mélanome métastatique, suggérant que la mise en place d'une réponse $T$ spécifique de ces néoantigènes pourrait être réactivée par l'élimination des Treg induite par l'ipilimumab. En utilisant les analyses de RNAseq de tumeurs de deux cohortes de patients atteints de mélanome métastatique traités par ipilimumab, Arce-Vargas et al. ont démontré que dans le cas de tumeurs ayant une forte charge mutationnelle, la proportion de réponses cliniques augmente fortement chez les patients porteurs du SNP RFc $\gamma$ IIIA V158F alors que ce SNP n'a pas d'impact lorsque les tumeurs présentent une faible charge mutationnelle [4]. L'expression du SNP RFc $\gamma$ IIA ${ }^{H 131 R}$ n'a pas d'impact puisqu'il est associé à une affinité accrue pour les hlgG2 et non les hlgGl.

\section{La déplétion des Treg dans l'environne-} ment tumoral après traitement par des Ac anti-CTLA-4 est controversée

Comme rapporté plus haut, il existe chez la souris un consensus [4,8] quant à la capacité des Ac anti-CTLA-4 à dépléter les Treg dans l'environnement tumoral, alors que très peu de données sont disponibles chez l'homme. Le groupe de Sharma [9], qui a récemment analysé le microenvironnement tumoral par immunohistochimie, dans des biopsies de tumeurs solides préet post-traitement avec l'ipilimumab ou le trémélimumab, indique que, contrairement aux données obtenues chez la souris, les Ac anti-CTLA-4 chez l'homme, quel que soit l'isotype, augmentent l'infiltration des $\mathrm{LT} C D 8^{+}$et $\mathrm{CD} 4^{+}$sans dépléter les Treg intra-tumoraux. Cependant, ces résultats 
sont en contradiction avec une autre étude chez des patients atteints de mélanome métastatique traitées par ipilimumab, qui démontre une forte réduction du nombre de Treg intra-tumoraux post-traitement chez les patients répondeurs [10].

L'absence de modulation des Treg dans la tumeur rapportée par Sharma et al. [9], peut résulter de l'analyse de biopsies réalisées à des temps tardifs posttraitement (> 5 semaines). En effet, des données de cinétique chez la souris démontrent que la déplétion des Treg est observée rapidement (2-5 jours) après traitement par les Ac anti-CTLA-4 [4]. Cette déplétion n'est sans doute plus détectable 5 à 8 semaines post-traitement du fait du recrutement de Treg périphériques non altérés. De plus, compte tenu du faible pourcentage de patients répondeurs, il aurait été intéressant de ségréger sur la réponse au traitement puisque cette diminution ne devrait être observée que chez les répondeurs.

\section{Perspectives}

L'ensemble de ces observations suggère que le développement de nouveaux Ac anti-CTLA-4, présentant une activité cytotoxique augmentée (comme les Ac de type $\mathrm{hlgGl} l_{\text {SDALIE, }}$ par exemple) pourrait être une stratégie intéressante pour améliorer le taux de réponse chez les patients traités. Malgré des résultats précliniques encourageants, une prudence est de mise quant aux effets secondaires d'une cytotoxicite accrue des anticorps anti-CTLA-4 puisque d'autres cellules telles que les LT CD 8 peuvent exprimer CTLA-4 après stimulation de leur récepteur (TCR). De plus, la détection du SNP RFc $\gamma$ IIIA ${ }^{\mathrm{V} 158 \mathrm{~F}}$ et l'analyse de la charge mutationnelle de la tumeur permettraient de sélectionner des patients plus aptes à répondre. L'expression des RFc $\gamma$ par l'infiltrat immunitaire dans l'environnement tumoral joue un rôle clef dans l'efficacité des thérapies si l'on souhaite éliminer une population délétère par ADCC. II serait donc judicieux de cibler des tumeurs chaudes infiltrées par des cellules immunitaires et d'analyser en détail les cellules accessoires présentes dans le microenvironnement tumoral. Enfin, il faut garder en tête que les ICP sont exprimés par des populations cellulaires autres que les Treg. Il est donc primordial de maîtriser la cinétique de traitement pour éviter une déplétion des LT effecteurs de la réponse anti-tumorale exprimant ces ICP après activation. $\diamond$

Treg cell contribution to anti-CTLA-4 therapeutic effect

\section{LIENS D'INTÉRÊT}

Les auteurs déclarent n'avoir aucun lien d'intérêt concernant les données publiées dans cet article.

\section{RÉFÉRENCES}

1. Brunet J-F, Denizot F, Luciani M-F, et al. A new member of the immunoglobulin superfamily-CTLA-4. Nature $1987 ; 328: 67$.

2. Ménétrier-Caux C, Curiel T, Faget J, et al. Targeting regulatory T cells. Targ Oncol $2012 ; 7: 15-28$.

3. Leach DR, Krummel MF, Allison JP. Enhancement of antitumor immunity by CTLA-4 blockade. Science $1996 ; 271: 1734-6$.

4. Arce Vargas F, Furness AJS, Litchfield K, et al. Fc Effector function contributes to the activity of human Anti-CTLA-4 antibodies. Cancer Cell 2018 ; 33 : 649-63.e4.

5. Du X, Tang F, Liu M, et al. A reappraisal of CTLA-4 checkpoint blockade in cancer immunotherapy. Cell Res 2018; $28: 416-32$

6. He M, Chai Y, Qi J, et al. Remarkably similar CTLA-4 binding properties of therapeutic ipilimumab and tremelimumab antibodies. Oncotarget $2017 ; 8$ : 67129-39.

7. Bruhns P. Properties of mouse and human IgG receptors and their contribution to disease models. Blood 2012; 119 : 5640-9.

8. Selby MJ, Engelhardt JJ, Quigley M, et al. Anti-CTLA-4 antibodies of $\lg G 2 a$ isotype enhance antitumor activity through reduction of intratumoral regulatory T cells. Cancer Immunol Res 2013 ; 1 : 32-42.

9. Sharma A, Subudhi SK, Blando J, et al. Anti-CTLA-4 immunotherapy does not deplete FOXP3 + regulatory T cells (Tregs) in human cancers. Clin Cancer Res 2019; $25: 1233-8$.

10. Romano E, Kusio-Kobialka M, Foukas PG, et al. Ipilimumab-dependent cell-mediated cytotoxicity of regulatory T cells ex vivo by nonclassical monocytes in melanoma patients. Proc Natl Acad Sci USA 2015 ; $112: 6140-5$

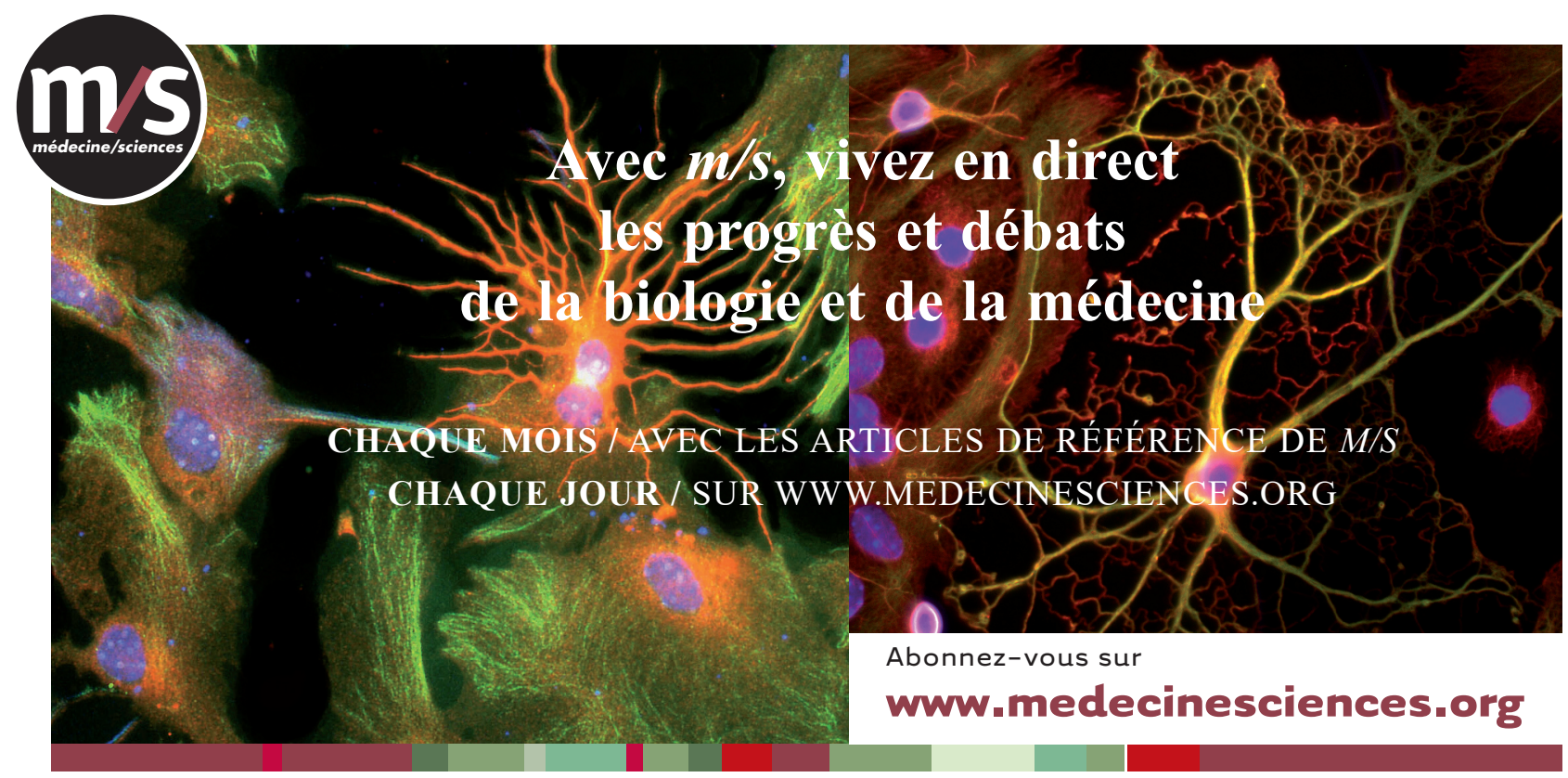

